\title{
Long term evolution of endothelial function during kidney transplantation
}

\author{
Clark Kensinger ${ }^{1}$, Aihua Bian², Meagan Fairchild ${ }^{3}$, Guanhua Chen², Loren Lipworth ${ }^{4}$, T. Alp Ikizler ${ }^{3}$ \\ and Kelly A. Birdwell ${ }^{3,5^{*}}$
}

\begin{abstract}
Background: Endothelial dysfunction is an important precursor to the development of atherosclerosis, and has been suggested to play a role in the increased cardiovascular risk in patients with end stage renal disease. Endothelial function improves rapidly following post kidney transplantation, but the long term change remains unclear. Hypothesizing that endothelial function would remain improved long term post kidney transplantation, we evaluated the longitudinal change of endothelial function, measured by flow-mediated dilation (FMD) of the brachial artery, from months 1 to 24 post transplantation. Given the previously reported association of fibroblast growth factor 23 (FGF-23) with endothelial dysfunction, we also examined changes in the association between FGF-23 levels and the change in FMD following kidney transplantation.
\end{abstract}

Methods: We performed a prospective cohort study of 149 kidney transplant recipients, measuring endothelial function by FMD at months 1, 12, and 24 post-transplant. FGF-23 levels were measured at months 1 and 24 post-transplant. Linear mixed effects models were used to assess both the unadjusted and adjusted outcomes.

Results: The cohort (mean age $49 \pm 13$ years) was $74 \%$ male and $75 \%$ white. The median FMD was $6.3 \%$ (IQR: 3.4, 10.2), $5.4 \%$ (IQR: 3.1, 8.5), and $5.6 \%$ (IQR: 3.5, 9.1) at 1, 12, and 24 months, respectively. After adjustment for covariates, compared to month 1, no change occurred in FMD at 12 months $(-0.66 \% ; 95 \% \mathrm{Cl}$ : -1 . $81 \%, 0.49 \% ; P=0.262)$ or 24 months $(-0.25 \% ; 95 \% \mathrm{Cl}:-1.76 \%, 1.26 \% ; P=0.746)$. FGF-23 decreased significantly over time $(P=0.024)$, but there was no significant association between FGF-23 and FMD $(P=0.799)$.

Conclusion: Endothelial function remained stable at 12 and 24 months from 1 month post-kidney transplant, indicating that the improved endothelial function seen with transplant is maintained up to 2 years post transplantation. There was also no significant association between FGF-23 and endothelial function following kidney transplantation.

Keywords: Cardiovascular disease, Cardiovascular risk factors, Endothelial dysfunction, Flow-mediated dilation, Kidney transplantation

\section{Background}

Patients with end stage renal disease (ESRD) requiring maintenance dialysis experience a high incidence and prevalence of cardiovascular disease, which is consequently a leading cause of mortality in this patient population [1]. Successful kidney transplantation is associated

\footnotetext{
* Correspondence: Kelly.Birdwell@vanderbilt.edu

${ }^{3}$ Division of Nephrology and Hypertension, Vanderbilt University Medical

Center, 1161 21st Avenue, S-3223 MCN, Nashville, TN 37232, USA

${ }^{5}$ Department of Medicine, Division of Nephrology and Hypertension,

Vanderbilt University Medical Center, 1161 21st Avenue, S-3223 MCN,

Nashville, TN 34232, USA

Full list of author information is available at the end of the article
}

with lower cardiovascular morbidity and mortality compared to patients who remain on the transplant wait list [2-4]. Despite improvement in traditional cardiovascular risk factors following kidney transplantation, the death rate from cardiovascular disease in patients with a functioning graft remains high compared to the general population [5]. Understanding the progression of unique cardiovascular risk factors associated with kidney transplantation may provide potential opportunities for risk modification leading to improved survival.

One marker of increased cardiovascular risk is impairment of endothelial function, which is an important 
precursor to the development of atherosclerosis. Impaired brachial artery endothelial function serves as a marker of underlying atherosclerotic risk and is a reliable surrogate for coronary endothelial function [6]. Several previous studies have reported impaired endothelial function in patients with chronic kidney disease [7-10]. Endothelial dysfunction is universally present in dialysis patients, and it can predict cardiovascular events in patients with kidney disease [7, 8, 11, 12]. Several studies have shown that endothelial function improves immediately post-transplant compared to pre-transplant [10, 13, 14]. However, no long term study of repeated endothelial function assessment has been performed in medical stable kidney transplant recipients.

A high concentration of circulating Fibroblast Growth Factor 23 (FGF-23) is also an independent risk factor for kidney disease progression, cardiovascular disease and mortality [15-21]. Fibroblast Growth Factor 23 is linked to endothelial dysfunction in patients with ESRD [19, 22-24] and FGF-23 levels have been shown to significantly decrease following successful kidney transplantation [15]. However, the degree to which endothelial function changes in conjunction with decreasing levels of FGF-23 following kidney transplant remains unclear.

Our aim was to evaluate endothelial dysfunction longitudinally, as measured by flow-mediated dilation (FMD), in a cohort of medically stable, kidney transplant recipients starting at 1 month post transplantation. In addition, we evaluated FGF-23 concentration following transplant to investigate a possible association between FGF-23 levels and changes in endothelial function. We hypothesized that long-term endothelial function would remain improved following successful kidney transplantation, and the longitudinal reduction in FGF-23 levels in the setting of a functioning allograft would be associated with improved endothelial function.

\section{Methods \\ Study design}

We conducted a single-center, prospective cohort study. De novo kidney transplant recipients were recruited from the Vanderbilt University Medical Center (VUMC) Renal Transplant Clinic from August 2009 through May 2013. Inclusion criteria included patients aged $\geq 18$ years who were undergoing or had recently undergone kidney transplantation. There were no exclusion criteria. Consent for participation in the study was obtained prior to discharge from the hospital following kidney transplant. Given the widely dispersed geographic area of patients on the kidney transplant waiting list at VUMC, obtaining a baseline FMD measurement prior to kidney transplantation was not logistically practical, thus patients were first evaluated at 1 month following kidney transplantation. Additional follow up visits were performed at
12 and 24 months. All patients gave informed consent and the VUMC Institutional Review Board approved the study protocol.

\section{Flow-mediated dilation protocol}

A single, licensed sonographer blinded to clinical details and previous FMD measurements of the study participants obtained the ultrasound images and performed the FMD study. The initial FMD was measured at 1 month following transplant. Follow up FMD examinations were performed at 12 and 24 months.

Flow-mediated dilation was performed as originally described by Celermajer and colleagues [25]. Endothelial function of the brachial artery was measured following ischemic reactive hyperemia to determine the resulting FMD. Each patient in the cohort was requested not to eat, drink (including alcohol, caffeine, etc.), or smoke for $12 \mathrm{~h}$ prior to the examination. The criteria to begin the study required a patient's systolic blood pressure to be stable within $5 \mathrm{mmHg}$ over several, separate recordings performed at two-minute intervals. A longitudinal image of the brachial artery was taken in the supine position at rest with a high-resolution ultrasound to obtain the baseline diameter (10.0 MHz linear array transducer, Philips iU 22, Andover, MA). A pneumatic tourniquet was inflated around the lower arm distal to the site at which the baseline brachial artery diameter was measured. The tourniquet was inflated to $250 \mathrm{mmHg}$ for a total time of $5 \mathrm{~min}$. Reactive hyperemia was measured after restoration of blood flow following the release of the pneumatic blood pressure cuff. Brachial artery diameter was measured at $60 \mathrm{~s}$ and $90 \mathrm{~s}$ following the release of the blood pressure cuff. Arterial diameter was measured from the media-adventitia interface.

The FMD studies were evaluated by one reviewer (CK) for consistency and accuracy of the brachial artery diameter measurements at the 60 and $90 \mathrm{~s}$ time points following the induction of hyperemia after the deflation of the pneumatic tourniquet.

\section{Flow-mediated dilation calculation}

Endothelium-dependent FMD was expressed as the percentage change of the brachial artery diameter from baseline to the diameter following reactive hyperemia (Post-test diameter-Baseline diameter)/Baseline diameter). The larger percent change of the two time points measured (60 or $90 \mathrm{~s}$ ) served as the reported FMD. An increase in FMD over time indicates improved endothelial function. An acceptable reproducibility during longitudinal evaluations is a mean difference of less than 2-3\% [26]. Therefore, we defined an improvement in FMD of more than $3 \%$ as necessary to detect a treatment benefit following kidney transplantation [27]. We 
also defined normal endothelial function as a FMD greater than $6.3 \%[28]$.

\section{Fibroblast growth factor-23 measurement}

Serum concentrations of FGF-23 were measured in duplicate after a single thaw of stored blood specimens using an intact FGF-23 ELISA kit (Immutopics, San Clemente, CA). Measurements in blood samples obtained at 1 month following kidney transplant served as the initial FGF-23 level. The longitudinal assessment of FGF-23 levels was performed on blood samples obtained at the 24-month follow-up. The coefficient of determination from the primary standard deviation curve for the FGF-23 measurements was 0.9999, indicating high reproducibility.

\section{Outcomes}

The primary outcome was defined as the change in FMD from the one-month post kidney transplant evaluation to the 12-month follow-up. Secondary outcomes include the change in FMD from 1 month to 24 months following kidney transplant, change in FMD from 12 months to 24 months following kidney transplant, and change in FGF-23 from 1 month to 24 months post-kidney transplant. We also examined the association between FGF-23 levels and the progression of FMD from 1 month to 24 months following kidney transplantation.

\section{Statistical analysis}

Descriptive statistics were presented as median with interquartile range (IQR) or mean with standard deviation (SD) for continuous variable and as percentages for categorical variables. We compared the differences in patients characteristics at month one between the patients who followed up at 12 months post transplant with those patients who did not return for evaluation using either Pearson's chi-square test (for categorical variables) or Wilcoxon rank sum test (for continuous variables). A linear mixed effects model with random intercepts was used to assess the change in FMD over time without and with adjustment for age, race, gender, smoking history (pack years), months on dialysis prior to transplant, cardiovascular disease, mean arterial pressure (MAP), diabetes, and estimated glomerular filtration rate (eGFR). Months on dialysis prior to transplant, eGFR, MAP, pack years, and age were included in the models as non-linear terms using restricted cubic splines. Cardiovascular disease was defined as a history of coronary artery disease, myocardial ischemia, reperfusion (coronary artery bypass or percutaneous stent), congestive heart failure, arrhythmia, stroke, or peripheral vascular disease.
A linear mixed effects model with random intercepts was used to examine the change in FGF-23 concentrations over time without and with adjustment for age, race, gender, cardiovascular disease, time on dialysis prior to transplant, and eGFR. In order to meet the normality assumption of the residuals, FGF-23 concentrations were natural logarithmic transformed. All the covariates for both models were chosen a priori. Analyses were performed using $\mathrm{R}$, version 3.1 .2 (http:// www.r-project.org/). The $5 \%$ significance level (2 sided) was used.

\section{Results}

\section{Patient demographic and other characteristics}

The study cohort included 149 patients who underwent evaluation at 1 month following kidney transplantation. Demographic and other patient characteristics are presented in Table 1. The cohort $(n=149$, mean age 49, SD $=39$ years) was $74 \%$ male and $75 \%$ White. The most common underlying etiology for ESRD was vascular disease (52\%; defined as ESRD due to diabetes- and/or hypertension-induced renal failure). Seventy-two patients (48\%) reported to be either current or former smokers, and 40 and $38 \%$ of the cohort had a history of cardiovascular disease and diabetes, respectively. The median time on dialysis prior to kidney transplant for the cohort was 20.3 months (IQR: 6.9, 47.2). Fifteen percent of the study cohort did not require hemodialysis prior to transplant. In regards to medications that may affect the progression of endothelial function, only $3 \%$ $(4 / 149)$ of the cohort were taking angiotensin converting enzymes inhibitors and angiotensin receptor blockers, $4 \%(6 / 149)$ were taking a vasodilator, $7 \%(11 / 149)$ were taking an alpha blocker, and $20 \%(29 / 149)$ were taking a statin. Throughout the two-year study period, there was one major cardiac event (myocardial infarction) that occurred between the 12 and 24-month follow up. There were six reported events of angina (three in the perioperative period prior to the 1 month evaluation, two events prior to the 12 month follow up, one event prior to the 24 month follow up) and one dysrhythmic event (occurred prior to the 12 month follow up).

Eighty-six patients had subsequent clinical follow-up at 12 months, and 44 patients at 24 months (Fig. 1). An analysis comparing characteristics of the patients who did and did not remain in the study through the 12month evaluation showed no statistically significant differences (For details, see Table 1 of the Supplemental Appendix). The two most common reasons (Fig. 1) for discontinuation prior to the 12-month visit were missing both the clinic appointment and ultrasound evaluation $(18 / 63,29 \%)$ or missing only the ultrasound evaluation (18/63, $29 \%)$. 
Table 1 Characteristics of the kidney transplant cohort at one-month post-transplantation

\begin{tabular}{ll}
\hline Characteristic & $N=149$ \\
\hline Age at transplant (years) & $49(40,59)$ \\
Race & \\
$\quad$ White & $112(75 \%)$ \\
Black & $34(23 \%)$ \\
Other & $3(2 \%)$ \\
Sex (male) & $111(74 \%)$ \\
BMl & $27.3(23.5,30.5)$
\end{tabular}

Tobacco use

Never
Current
Former
Pack Years
Cardiovascular disease ${ }^{a}$
Diabetes
Hypertension
Hyperlipidemia
Months on Dialysis pre-transplant
Primary cause of end-stage renal disease
Vascular Disease
Glomerular Disease
Tubulointerstitial Disease
Cystic Disease
Structure Disease
Renal Neoplasia
Other

$77(52 \%)$

$14(9 \%)$

$58(39 \%)$

$11.5(3,24.0)$

$59(40 \%)$

$56(38 \%)$

$142(95 \%)$

$83(56 \%)$

$20.3(6.9,47.2)$

$78(52 \%)$

$31(21 \%)$

$9(6 \%)$

$19(13 \%)$

$1(1 \%)$

$1(1 \%)$

$10(7 \%)$

Estimated Glomerular Filtration Rate $\left(\mathrm{ml} / \mathrm{min} / \mathrm{m}^{2}\right)$

$58(48,69)$

$95.7(87.3,103.7)$

Mean Arterial Pressure $(\mathrm{mm} \mathrm{Hg})$

Induction Immunosuppression

Alemtuzumab

$134(90 \%)$

Basiliximab

$14(9 \%)$

Anti-Thymocyte Globulin

$1(1 \%)$

Maintenance Immunosuppression

Tacrolimus

$146(98 \%)$

Mycophenolate

$143(96 \%)$

Prednisone

$81(54 \%)$

Anti-Hypertensive Medication

Alpha Blockers

$11(7 \%)$

Beta Blockers

$88(59 \%)$

Calcium Channel Blockers

77 (52\%)

ACE $^{\mathrm{b}}$ Inhibitors

$4(3 \%)$

$4(3 \%)$

Angiotensin Receptor Blocker

Vasodilators
Table 1 Characteristics of the kidney transplant cohort at one-month post-transplantation (Continued)

\begin{tabular}{ll}
\hline Statin Therapy & $29(20 \%)$ \\
Slow Graft Function & $23(16 \%)$ \\
Delayed Graft Function & $8(5 \%)$
\end{tabular}

Values expressed as median $\left(25^{\text {th }}, 75^{\text {th }}\right.$ percentiles) or number (percent) ${ }^{a}$ Cardiovascular disease includes coronary artery disease, myocardial infarction, coronary artery bypass grafting, percutaneous coronary intervention, congestive heart failure, arrhythmia, stroke, and peripheral vascular disease

${ }^{\mathrm{b}}$ Angiotensin Converting Enzyme

\section{Primary outcome}

The median FMD at one-month post-kidney transplant for the cohort was $6.3 \%$ (IQR: 3.4-10.2) and at 12 months was $5.4 \%$ (IQR: 3.1-8.5) (Table 2). Based on our definition of a normal FMD (>6.3\%), $52 \%$ $(77 / 149)$ of the population had normal endothelial function at 1 month and $49 \%(42 / 86)$ at 12 months. In the unadjusted model, FMD remained stable over time (overall $P=0.622$ ). Compared to month one, FMD numerically decreased by $0.56 \%$ (95 \% CI: $-1.70,0.57, P=0.332$ ) at month 12. In the adjusted model controlling for age, race, gender, smoking history (pack years), months on dialysis prior to transplant, cardiovascular disease, MAP, diabetes and eGFR, FMD remained stable over time (overall $P=0.532$ ). Compared to month one in the adjusted

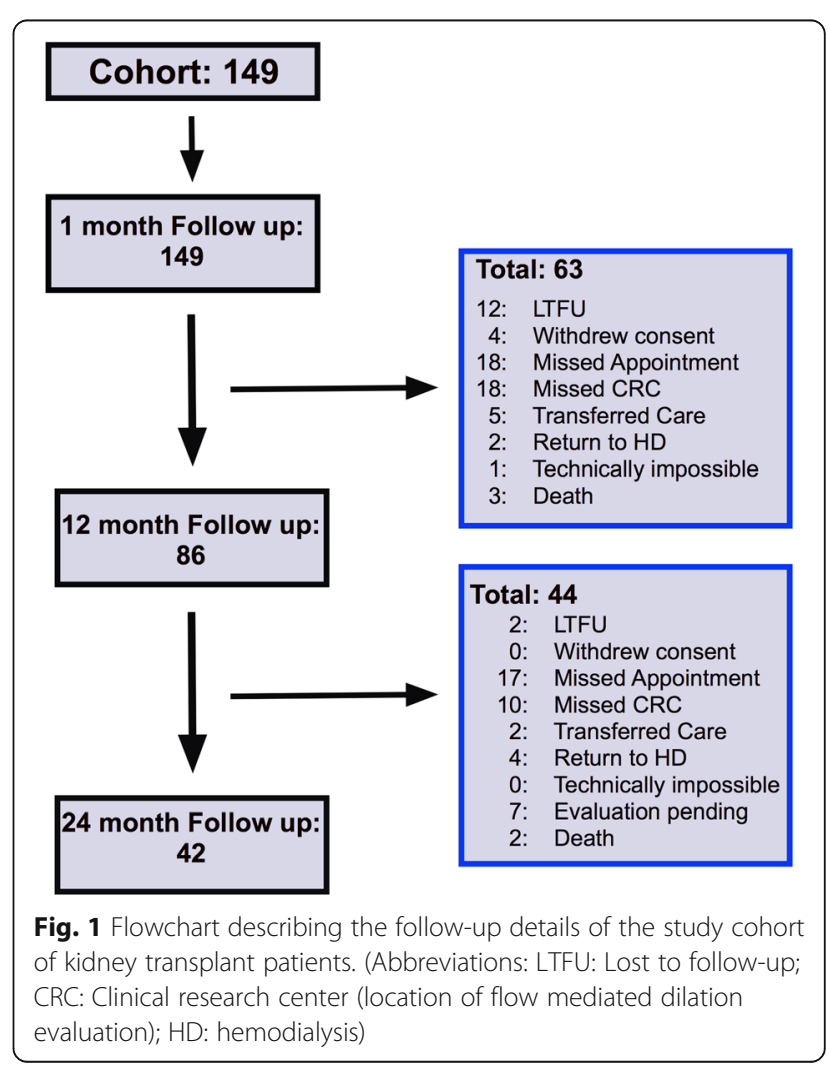


Table 2 Changes in flow mediated dilation and other clinical characteristics during the study period

\begin{tabular}{ll}
\hline Parameters & \\
\hline Flow Mediated Dilatation (percentage) & $6.3(3.4,10.2)$ \\
Month 1 & $5.4(3.1,8.5)$ \\
Month 12 & $5.6(3.5,9.1)$ \\
Month 24 & \\
Body Mass Index $\left(\mathrm{kg} / \mathrm{m}^{2}\right)$ & $27.3(23.5,30.5)$ \\
Month 1 & $29.2(26.2,34.0)$ \\
Month 12 & $31.0(26.2,33.4)$ \\
Month 24 & $58(48,69)$ \\
Estimated Glomerular Filtration Rate $\left(\mathrm{ml} / \mathrm{min} / 1.73^{*} \mathrm{~m}^{2}\right)$ \\
Month 1 & $59(47,71)$ \\
Month 12 & $57(45,69)$ \\
Month 24 & \\
Mean Arterial Pressure (mmHg) & $95.7(87.3,103.7)$ \\
Month 1 & $96.3(90.8,103.7)$ \\
Month 12 & $98.7(92.5,104.7)$ \\
Month 24 &
\end{tabular}

Values expressed as median $\left(25^{\text {th }}, 75^{\text {th }}\right.$ percentiles $)$

model, FMD numerically decreased by $0.66 \%$ at month 12, but this was not statistically significant (95 \% CI: $-1.81,-0.49, P=0.262$ ) (Table 3). Females had significantly increased FMD compared to males $(P=0.031)$, and FMD decreased significantly with age $(P=0.038)$.

Table 3 Multivariable linear regression model of flow mediated dilation in kidney transplant recipients

\begin{tabular}{lllll}
\hline Covariate & Effect & S.E. & $95 \% \mathrm{Cl}^{\mathrm{a}}$ & $P$ \\
Predictors of FMD & & & & \\
\hline Age at transplant & -0.06 & 0.58 & $-1.20,1.08$ & 0.038 \\
Race (reference: white) & -0.94 & 0.87 & $-2.63,0.76$ & 0.382 \\
Sex (reference: males) & 1.71 & 0.79 & $0.15,3.26$ & 0.031 \\
Diabetes & -1.27 & 0.75 & $-2.74,0.20$ & 0.091 \\
Cardiovascular Disease ${ }^{b}$ & -0.87 & 0.76 & $-2.35,0.62$ & 0.252 \\
Pack Years & -0.44 & 0.47 & $-1.36,0.48$ & 0.150 \\
Glomerular filtration rate & -0.47 & 0.43 & $-1.32,0.37$ & 0.362 \\
Mean arterial pressure & -0.42 & 0.39 & $-1.19,0.34$ & 0.976 \\
Time on dialysis & -0.45 & 0.8 & $-2.01,1.1$ & 0.984 \\
FMD 1 month -> 12 months & -0.66 & 0.59 & -1.81 .0 .49 & 0.262 \\
FMD 1 month - > 24 months & -0.25 & 0.77 & $-1.76,1.26$ & 0.746 \\
FMD 12 months $->$ 24 months & 0.41 & 0.81 & $-1.19,2.01$ & 0.614 \\
\hline
\end{tabular}

${ }^{\mathrm{a}} 95 \%$ Confidence Interval: Lower bound, Upper bound

${ }^{b}$ Cardiovascular disease includes coronary artery disease, myocardial infarction, coronary artery bypass grafting, percutaneous coronary intervention,

congestive heart failure, arrhythmia, stroke, and peripheral vascular disease

\section{Secondary outcomes} FMD at 24 months

The median FMD for the cohort at 24 months was $5.6 \%$ (IQR: 3.5-9.1) (Fig. 2). Based on our definition of a normal FMD (>6.3 \%), $50 \%(22 / 44)$ of the population had normal endothelial function at 24 months. In the adjusted model, FMD remained stable from 1 month to 24 months $(-0.25 \%, 95 \% \mathrm{CI}:-1.76,1.26, P=0.746)$ and from 12 months to 24 months $(0.41 \%, 95 \%$ CI: -1.19 , 2.01, $P=0.614)$ post-transplantation.

\section{FGF-23 levels}

Forty-five patients were included in the analysis that measured FGF-23 at 1 month and 24 months follow-up after kidney transplantation. Fibroblast Growth Factor 23 decreased significantly over time $(P=0.02)$ (Fig. 3). At month 24, FGF-23 decreased $36 \%$ (95 \% CI: $7 \%$, $56 \%, P=0.024)$ compared to month one.

\section{Flow-mediated dilation and FGF-23 level}

Adjusting for age, race, gender, cardiovascular disease, time on dialysis prior to transplant and eGFR, there was no statistically significant relationship between FGF-23 and FMD following kidney transplant $(P=0.799)$. With one interquartile range ( 100 picograms per milliliters) decrease in FGF-23, FMD decreased by $0.11 \%$ (95 \% CI: $-0.93,0.71, P=0.799)$ over time from 1 month to 24 months post transplant. In addition, there was no significant association between FGF-23 and FMD at month one $(P=0.959)$ or month $24(P=0.699)$.

\section{Discussion}

Endothelial dysfunction is characterized by an impaired vasodilation in response to changes in the local hemodynamic forces and vasoactive substances and can initiate the atherosclerotic pathway [29]. Since atherosclerotic disease initiated by endothelial dysfunction is a frequent cause of cardiovascular morbidity and mortality in patients with ESRD, studying changes in endothelial function by FMD may provide important information to evaluate potential interventions to address risk modification for cardiovascular disease following kidney transplantation [29]. Therefore, the aim of this study was to evaluate the long term evolution of endothelial function in kidney transplantation. We report that the improved endothelial function observed immediately post transplant is well maintained up to 2 years post transplantation. In multivariate models, only age and sex were predictors of FMD.

Endothelial dysfunction is universally present in dialysis patients, and previous studies have demonstrated a favorable change in FMD comparing pre and posttransplantation. Kocak et al demonstrated that FMD significantly improved 14 days after kidney transplant 


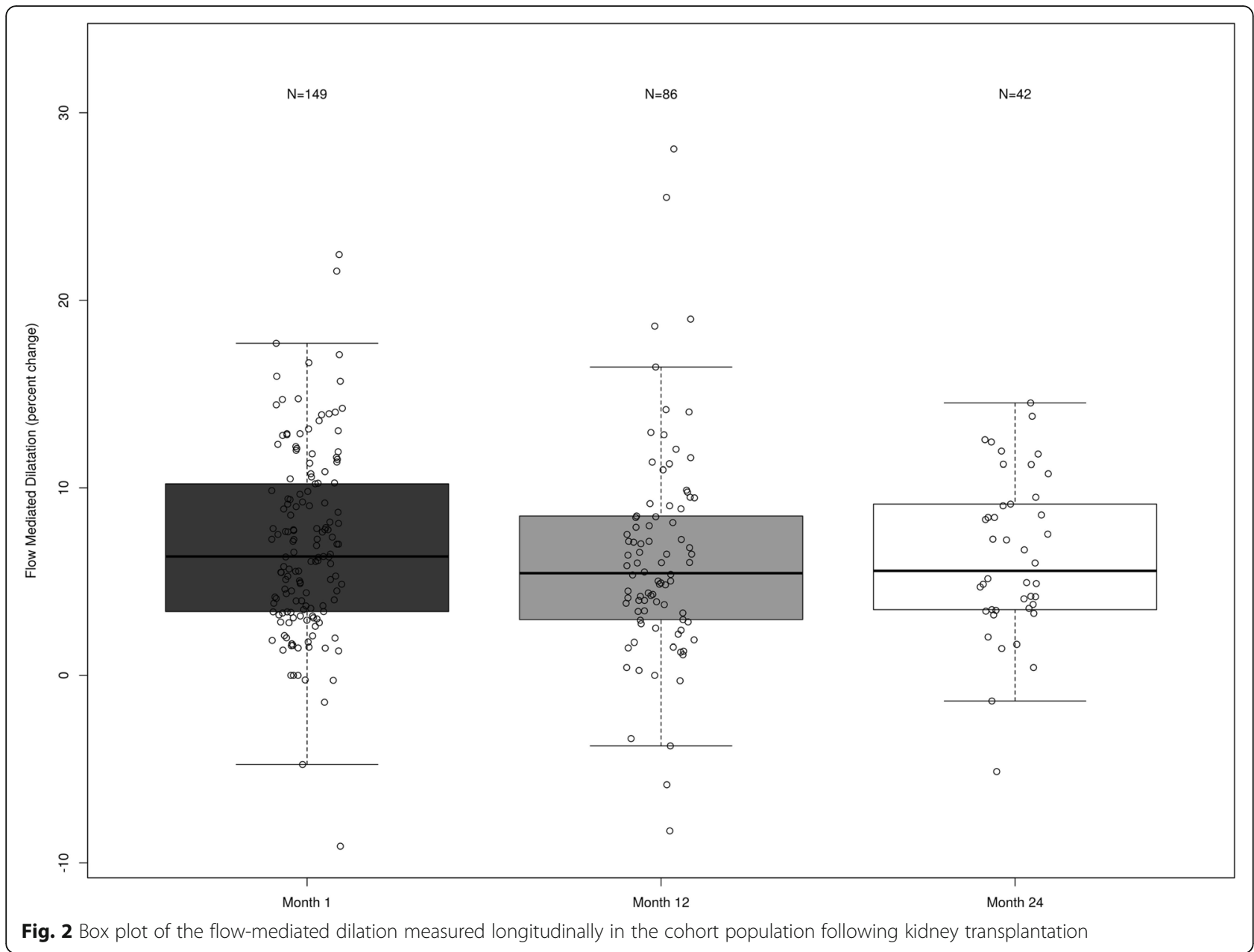

(6.69 \% vs. $10.50 \%, P<0.001)$ [14]. In that study, 30 chronic hemodialysis patients with an average age of 38 years were included following living donation kidney transplant [14]. Oflaz et al evaluated endothelial function in 22 ESRD patients with an average age of 34 years and body mass index (BMI) of 23.1 kilograms $/$ meter $^{2}(\mathrm{~kg} /$ $\mathrm{m}^{2}$ ) [13], and reported a significant improvement in FMD from pre-transplant $(6 \pm 3.7 \%)$ versus 6 months $(8.3 \pm 2.3 \%(P=<0.001))$ and 12 months following transplant (12 months: $12.1 \pm 3.6 \%(P=<0.001))$ [13]. Yilmaz et al showed that endothelium-dependent vasodilation improved within 6 months following kidney transplant in 161 patients with an average age of 31 and average BMI of $25.3 \mathrm{~kg} / \mathrm{m}^{2}$ [10]. Flow mediated dilation prior to transplant was $5.2 \pm 0.8$ and 6 months following transplant was $6.6 \pm 0.7(P=<0.001)[10]$.

Our study, in contrast, examines the longer-term effect of kidney transplantation on endothelial function, showing that FMD remains relatively stable from 1 month to 24 months post transplant. The patients in our cohort were initially evaluated at 1 month post transplant, a time when many of the biochemical abnormalities related to uremia have corrected and complications related to rejection or infection are rare.

Strengths of our study include the size of our cohort and duration of longitudinal follow-up. Moreover, the population in our study is older and includes a substantial proportion of patients with cardiovascular disease or diabetes, compared to the previously mentioned studies that were conducted among select groups of relatively young transplant recipients. Thus, a potential explanation for the lack of ongoing improvement in FMD in our study is the high cardiovascular risk profile of the transplant recipients. However, our study population is more reflective of the ESRD and transplant recipient population in the United States and Europe, which improves the generalizability of our findings compared to prior reports. Another strength of our study was that a single sonographer performed the FMD study in the entire cohort, which improves the reliability of the results. The advantage of using brachial artery FMD as a surrogate for coronary artery endothelial health is that the test is noninvasive with reproducible results in the same patient over time when performed by the same examiner. 


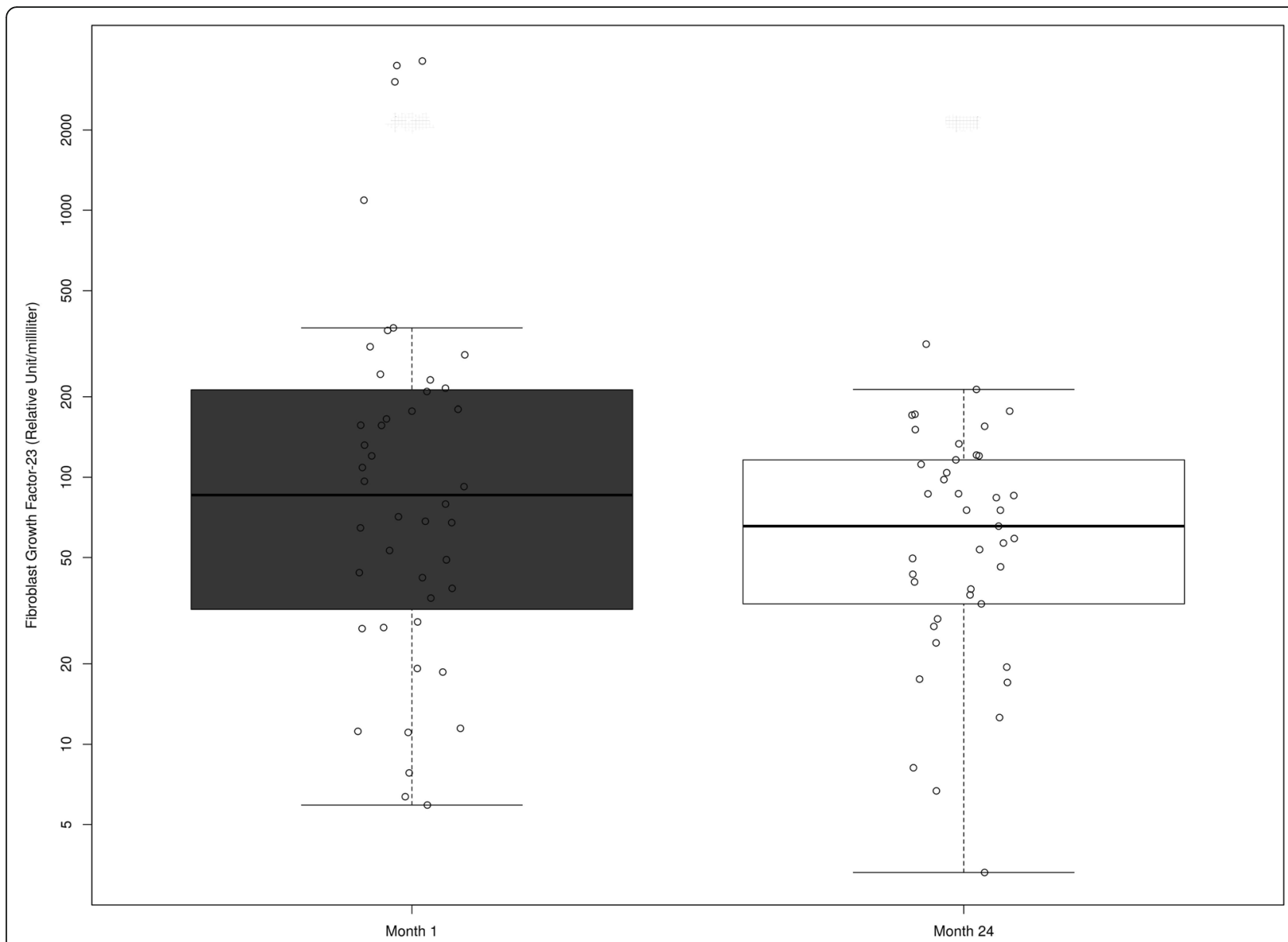

Fig. 3 Box plot of the fibroblast growth factor 23 levels measured longitudinally in the cohort population following kidney transplantation

A limitation of the study is the lack of a pre-transplant FMD measurement. There are reports that note FMD improves rapidly with favorable modification of cardiovascular risk factors. In the prospective, randomized RECIFE trial, FMD was shown to significantly improve in just 6 weeks $(4.93+/-0.81 \%$ to $7.0+/-0.79 \%(P=$ $0.02)$ ) in a high cardiovascular risk patient population (i.e. acute myocardial infarction or unstable angina) with hyperlipidemia who took pravastatin compared to placebo [30]. This study suggests that endothelial function rapidly improves with interventions that favorably modify cardiovascular risk, such as kidney transplantation. When compared to our study, the report by Yilmaz et al. [1] provides further insight into the potential improvement in FMD during the early post-operative period. The average FMD at 1 month after transplant in our study was $6.4 \%$ which is similar to the FMD at 6 months following transplant $(6.6 \%)$ in the Yilmaz et al report; however, this measurement corresponded to a $27 \%$ increase in FMD when compared to the pre-transplant FMD of $5.2 \%$. Without a pre-transplant FMD measurement, the immediate changes in endothelial function within the early post-operative period were not captured in our study design compared the prior reports $[10,13,14]$ where the baseline FMD measurement was based on pretransplant evaluations. However, we feel our study does provide additional information on the long-term effect of kidney transplantation on endothelial function. It is interesting to note endothelial function as measured by FMD remains stable during the first 2 years post transplantation despite the ongoing adverse metabolic effects from transplant immunosuppression and possible decline in kidney function.

Fibroblast Growth Factor-23 has been shown to be significantly elevated in patients with chronic kidney disease as FGF-23 metabolism and clearance are modified [31, 32], which subsequently improves following kidney transplantation [15]. Several studies have shown a link between elevated FGF-23 levels and increased cardiovascular risk. In a prospective study of 984 kidney transplant patients, Wolf et al showed that elevated FGF-23 levels post-operatively were independently associated with increased mortality and allograft loss [15]. Elevated FGF-23 levels have been correlated with impaired 
endothelial function in patients [19, 22-24]. Mirza et al demonstrated an association between higher FGF-23 levels and impaired vasoreactivity, as well as, a correlation between arterial stiffness with patients with renal dysfunction and higher FGF-23 levels [24]. Yilmaz et al reported an independent correlation of high FGF-23 and impaired FMD in patients with stage III and IV renal failure (20). The relationship between decreased FGF-23 concentrations and endothelial function following kidney transplantation is not well studied, but one report showed in 161 patients that endothelium-dependent vasodilation improved in the 6 months following kidney transplant, which corresponded to a significant decrease in FGF-23 levels [10]. In our study, there was no significant association between FGF-23 levels and FMD over 24 months following kidney transplant despite a significant reduction in FGF-23 concentration over the twoyear period. The difference in outcomes between the two studies could be a result of the shorter follow up time in the Yilmaz et al report (6 months versus 24 months, respectively) and, as previously stated, the dissimilarity in the demographic profiles.

Further limitations of our study include the high percentage of patients who did not complete the follow up. A sensitivity analysis did not show any significant demographic or clinical differences between patients who did not return for the 12 month follow up compared to patients who completed the study, suggesting that any bias is likely to be minimal, though in characteristics not examined cannot be ruled out. Another limitation of our study is that measurements for maximal FMD were obtained at set time points of 60 and $90 \mathrm{~s}$. Without a continuous evaluation, the maximum diameter change secondary to reactive hyperemia may not have been captured with these set time point measurements. In addition, FMD can be affected by temperature, foods, drugs, sympathetic stimuli, environmental factors, and patient's positions, which can vary over time at each evaluation. The study protocol attempted to minimize this variation by asking all patients to not consume tobacco, food or liquids for $8 \mathrm{~h}$ prior to the evaluation. In addition, cardiovascular medication such as angiotensin converting enzyme inhibitors and statins that could affect endothelial function were not controlled for in the study cohort.

\section{Conclusions}

In a single center, prospective study of medically stable, kidney transplant recipients who were initially evaluated at 1 month following surgery, FMD remained stable over a 24 month follow up period. Consistent with previous work, FGF-23 levels significantly decreased following kidney transplant, however this decrease was not associated with improvement in FMD. The stable FMD in our study cohort suggests that the improvement in endothelial function is maintained up to 2 years post transplantation. However, the lack of continued improvement in endothelial function may contribute to the persistently elevated cardiovascular risk in transplant patients compared to the general population despite improved renal function. Further prospective research regarding endothelial function following kidney transplant would provide important insight into possible mechanisms whereby transplantation impacts cardiovascular health, which would allow interventions aimed at the prevention and reversibility of cardiovascular diseases.

\begin{abstract}
Abbreviations
BMI: Body mass index; eGFR: Estimated glomerular filtration rate; ESRD: End stage renal disease; FGF-23: Fibroblast growth factor; FMD: Flow mediated dilation; IQR: Interquartile range; $\mathrm{kg} / \mathrm{m}^{2}$ : Kilograms/meter ${ }^{2}$; MAP: Mean arterial pressure; SD: Standard deviation; VUMC: Vanderbilt university medical center
\end{abstract}

\section{Acknowledgements}

The authors have none to report.

\section{Funding}

1) UL1TR000445 (Ikizler) from the National Center for Advancing Translational Sciences

2) S10RR027033 (Ikizler) from the National Center for Research Resources 3) K24 DK62849 (Ikizler) and P30 DK079341 from the National Institute of Diabetes, Digestive and Kidney Diseases

4) R01 HL070938 (Ikizler) from the National Heart, Lung, and Blood Institute 5) K23 GM100183 (Birdwell) from the National Institute of General Medical Sciences

6) T32DK007569 (Kensinger) from the National Institute of Diabetes, Digestive and Kidney Diseases

\section{Availability of data and materials}

The data will not be publicly available but unidentifiable data can be provided in full if requested by the editors, funding agencies and any requests for meta-analyses.

\section{Authors' contributions}

CDK participated in research design, writing of the paper, performance of the research, and data analysis, and editing of the final manuscript. $A B$ participated in research design, writing of the paper, data analysis, contributed to analytic tools, and editing of the final manuscript. MF participated in data acquisition, performance of the research, editing and approval of the final manuscript. GC participated in research design, writing of the paper, data analysis, contributed to analytic tools, editing and approval of the final manuscript. LL-E participated in research design, editing of the manuscript and approval of the article. TAI participated in research design, editing of the manuscript, and approval of the article. KB participated in research design, writing of the paper, performance of the research, and data analysis, and editing of the final manuscript. All authors read and approved the final manuscript.

\section{Competing interest}

The authors declare that they have no competing interests.

\section{Consent for publication}

As only summary congregated data are presented, consent for publication of patients is not applicable.

\section{Ethics approval and consent to participate}

The Vanderbilt University Medical Center Institutional Review Board, who oversees human subjects protection and ethical oversight, approved the study protocol. All patients gave signed informed consent. 


\section{Author details}

'Department of Surgery, Vanderbilt University Medical Center, 1161 21st Avenue South, D4313 MCN, Nashville, TN 37232, USA. ${ }^{2}$ Department of Biostatistics, Vanderbilt University Medical Center, 2525 West End Avenue, Suite 11000, Nashville, TN 37203, USA. ${ }^{3}$ Division of Nephrology and Hypertension, Vanderbilt University Medical Center, 1161 21st Avenue, S-3223 MCN, Nashville, TN 37232, USA. " Division of Epidemiology, Department of Medicine, Vanderbilt University Medical Center, 2525 West End, Suite 600, Nashville, TN 37203, USA. ${ }^{5}$ Department of Medicine, Division of Nephrology and Hypertension, Vanderbilt University Medical Center, 116 21st Avenue, S-3223 MCN, Nashville, TN 34232, USA.

\section{Received: 24 April 2016 Accepted: 11 October 2016}

\section{Published online: 22 October 2016}

\section{References}

1. US Renal Data System: USRDS 2010 Annual Data Report: Atlas of Chronic Kidney Disease \& End-Stage Renal Disease in the United States. National Institutes of Health, National Institute of Diabetes and Digestive and Kidney Diseases. 2014; Volume 2

2. Wolfe RA, Ashby VB, Milford EL, Ojo AO, Ettenger RE, Agodoa LY, et al, Comparison of mortality in all patients on dialysis, patients on dialysis awaiting transplantation, and recipients of a first cadaveric transplant. N Engl J Med. 1999:341(23):1725-30.

3. Meier-Kriesche HU, Schold JD, Srinivas TR, Reed A, Kaplan B. Kidney transplantation halts cardiovascular disease progression in patients with end-stage renal disease. Am J Transplant. 2004;4(10):1662-8.

4. Pilmore H, Dent H, Chang S, McDonald SP, Chadban SJ. Reduction in cardiovascular death after kidney transplantation. Transplantation. 2010;89(7):851-7.

5. United States Renal Data System. 2014 annual data report: An overview of the epidemiology of kidney disease in the United States. National Institutes of Health, National Institute of Diabetes and Digestive and Kidney Diseases. 2014

6. Anderson TJ, Uehata A, Gerhard MD, Meredith IT, Knab S, Delagrange D, et al. Close relation of endothelial function in the human coronary and peripheral circulations. J Am Coll Cardiol. 1995:26(5):1235-41.

7. Thambyrajah J, Landray MJ, McGlynn FJ, Jones HJ, Wheeler DC, Townend $\mathrm{JN}$. Abnormalities of endothelial function in patients with predialysis renal failure. Heart. 2000;83(2):205-9.

8. Recio-Mayoral A, Banerjee D, Streather C, Kaski JC. Endothelial dysfunction, inflammation and atherosclerosis in chronic kidney disease-a cross-sectional study of predialysis, dialysis and kidney-transplantation patients. Atherosclerosis. 2011:216(2):446-51.

9. Reffelmann T, Krebs A, Ittermann T, Empen K, Hummel A, Dorr M, et al. Mild renal dysfunction as a non-traditional cardiovascular risk factor?-Association of cystatin C-based glomerular filtration rate with flow-mediated vasodilation. Atherosclerosis. 2010;211(2):660-6.

10. Yilmaz MI, Sonmez A, Saglam M, Yaman H, Kilic S, Turker T, et al. Longitudinal analysis of vascular function and biomarkers of metabolic bone disorders before and after renal transplantation. Am J Nephrol. 2013;37(2):126-34

11. Stam F, van Guldener C, Becker A, Dekker JM, Heine RJ, Bouter LM, et al. Endothelial dysfunction contributes to renal function-associated cardiovascular mortality in a population with mild renal insufficiency: the Hoorn study. J Am Soc Nephrol. 2006;17(2):537-45.

12. Kuczmarski JM, Darocki MD, DuPont JJ, Sikes RA, Cooper CR, Farquhar WB, et al. Effect of moderate-to-severe chronic kidney disease on flow-mediated dilation and progenitor cells. Exp Biol Med (Maywood). 2011:236(9):1085-92.

13. Oflaz H, Turkmen A, Turgut F, Pamukcu B, Umman S, Ucar A, et al. Changes in endothelial function before and after renal transplantation. Transpl Int 2006;19(4):333-7.

14. Kocak H, Ceken K, Yavuz A, Yucel S, Gurkan A, Erdogan O, et al. Effect of renal transplantation on endothelial function in haemodialysis patients. Nephrol Dial Transplant. 2006;21(1):203-7.

15. Wolf M, Molnar MZ, Amaral AP, Czira ME, Rudas A, Ujszaszi A, et al. Elevated fibroblast growth factor 23 is a risk factor for kidney transplant loss and mortality. J Am Soc Nephrol. 2011:22(5):956-66.

16. Gutierrez OM, Mannstadt M, Isakova T, Rauh-Hain JA, Tamez H, Shah A, et al. Fibroblast growth factor 23 and mortality among patients undergoing hemodialysis. N Engl J Med. 2008;359(6):584-92.
17. Gutierrez OM, Januzzi JL, Isakova T, Laliberte K, Smith K, Collerone G, et al. Fibroblast growth factor 23 and left ventricular hypertrophy in chronic kidney disease. Circulation. 2009;119(19):2545-52.

18. Fliser D, Kollerits B, Neyer U, Ankerst DP, Lhotta K, Lingenhel A, et al. Fibroblast growth factor 23 (FGF23) predicts progression of chronic kidney disease: the mild to moderate kidney disease (MMKD) study. J Am Soc Nephrol. 2007;18(9):2600-8.

19. Mirza MA, Hansen T, Johansson L, Ahlstrom H, Larsson A, Lind L, et al. Relationship between circulating FGF23 and total body atherosclerosis in the community. Nephrol Dial Transplant. 2009;24(10):3125-31.

20. Dhingra R, Sullivan LM, Fox CS, Wang TJ, D'Agostino Sr RB, Gaziano JM, et al. Relations of serum phosphorus and calcium levels to the incidence of cardiovascular disease in the community. Arch Intern Med. 2007:167(9):879-85.

21. Kestenbaum B, Sampson JN, Rudser KD, Patterson DJ, Seliger SL, Young B, et al. Serum phosphate levels and mortality risk among people with chronic kidney disease. J Am Soc Nephrol. 2005;16(2):520-8.

22. Yilmaz MI, Sonmez A, Saglam M, Yaman H, Kilic S, Demirkaya E, et al. FGF-23 and vascular dysfunction in patients with stage 3 and 4 chronic kidney disease. Kidney Int. 2010;78(7):679-85.

23. Yilmaz MI, Sonmez A, Saglam M, Yaman H, Kilic S, Eyileten T, et al. Comparison of calcium acetate and sevelamer on vascular function and fibroblast growth factor 23 in CKD patients: a randomized clinical trial. Am J Kidney Dis. 2012;59(2):177-85.

24. Mirza MA, Larsson A, Lind L, Larsson TE. Circulating fibroblast growth factor-23 is associated with vascular dysfunction in the community. Atherosclerosis. 2009:205(2):385-90.

25. Celermajer DS, Sorensen KE, Gooch VM, Spiegelhalter DJ, Miller OI, Sullivan ID, et al. Non-invasive detection of endothelial dysfunction in children and adults at risk of atherosclerosis. Lancet. 1992;340(8828):1111-5.

26. Corretti MC, Anderson TJ, Benjamin EJ, Celermajer D, Charbonneau F, Creager MA, et al. Guidelines for the ultrasound assessment of endothelialdependent flow-mediated vasodilation of the brachial artery: a report of the international brachial artery reactivity task force. J Am Coll Cardiol. 2002;39(2):257-65

27. Sorensen KE, Celermajer DS, Spiegelhalter DJ, Georgakopoulos D, Robinson J, Thomas $\mathrm{O}$, et al. Non-invasive measurement of human endothelium dependent arterial responses: accuracy and reproducibility. Br Heart J. 1995;74(3):247-53

28. Fathi $R$, Haluska B, Isbel N, Short L, Marwick TH. The relative importance of vascular structure and function in predicting cardiovascular events. J Am Coll Cardiol. 2004;43(4):616-23

29. Verma $S$, Anderson TJ. Fundamentals of endothelial function for the clinical cardiologist. Circulation. 2002;105(5):546-9.

30. Dupuis J, Tardif JC, Cernacek P, Theroux P. Cholesterol reduction rapidly improves endothelial function after acute coronary syndromes. The RECIFE (reduction of cholesterol in ischemia and function of the endothelium) trial. Circulation. 1999;99(25):3227-33.

31. Mathew JS, Sachs MC, Katz R, Patton KK, Heckbert SR, Hoofnagle AN, et al. Fibroblast growth factor-23 and incident atrial fibrillation: the multi-ethnic study of atherosclerosis (MESA) and the cardiovascular health study (CHS). Circulation. 2014;130(4):298-307.

32. Sanchez Fructuoso Al, Maestro ML, Perez-Flores I, Valero R, Rafael S, Veganzones $\mathrm{S}$, et al. Serum level of fibroblast growth factor 23 in maintenance renal transplant patients. Nephrol Dial Transplant. 2012;27(11):4227-35. 